Research article

\title{
Bidirectional transcription of a novel chimeric gene mapping to mouse chromosome $\mathbf{Y q}$ Peter JI Ellis ${ }^{\dagger}$, Lydia Ferguson ${ }^{\dagger}$, Emily J Clemente and Nabeel A Affara*
}

Address: Mammalian Molecular Genetics Group, University of Cambridge Department of Pathology, Tennis Court Rd., Cambridge, CB2 1QP, UK Email: Peter JI Ellis - pjie2@cam.ac.uk; Lydia Ferguson - If268@cam.ac.uk; Emily J Clemente - emilyclemente@hotmail.com; Nabeel A Affara* - na@mole.bio.cam.ac.uk

* Corresponding author †Equal contributors

Published: 24 September 2007

BMC Evolutionary Biology 2007, 7:17| doi:|0.||86/|47|-2|48-7-|7|
Received: 17 April 2007

Accepted: 24 September 2007

This article is available from: http://www.biomedcentral.com/l47/-2/48/7/I7I

(c) 2007 Ellis et al; licensee BioMed Central Ltd.

This is an Open Access article distributed under the terms of the Creative Commons Attribution License (http://creativecommons.org/licenses/by/2.0), which permits unrestricted use, distribution, and reproduction in any medium, provided the original work is properly cited.

\begin{abstract}
Background: The male-specific region of the mouse $Y$ chromosome long arm (MSYq) contains three known highly multi-copy X-Y homologous gene families, Ssty I/2, Sly and Asty. Deletions on MSYq lead to teratozoospermia and subfertility or infertility, with a sex ratio skew in the offspring of subfertile MSYqdel males

Results: We report the highly unusual genomic structure of a novel MSYq locus, Orly, and a diverse set of spermatid-specific transcripts arising from copies of this locus. Orly is composed of partial copies of Sstyl, Asty and Sly arranged in sequence. The Sstyl- and Sly-derived segments are in antisense orientation relative to each other, leading to bi-directional transcription of Orly. Genome search and phylogenetic tree analysis is used to determine the order of events in mouse $\mathrm{Yq}$ evolution. We find that Orly is the most recent gene to arise on $\mathrm{Yq}$, and that subsequently there was massive expansion in copy number of all Yq-linked genes.

Conclusion: Orly has an unprecedented chimeric structure, and generates both "forward" (Orly) and "reverse" (Orlyos) transcripts arising from the promoters at each end of the locus. The region of overlap of known Orly and Orlyos transcripts is homologous to Sly intron 2. We propose that Orly may be involved in an intragenomic conflict between mouse $X$ and $Y$ chromosomes, and that this process underlies the massive expansion in copy number of the genes on MSYq and their $X$ homologues.
\end{abstract}

\section{Background}

The mammalian Y chromosome is constitutively haploid, restricted to males, and subject to ongoing genetic deterioration due to lack of recombinational exchange with a homologous partner. Set against this, however, there is strong evolutionary drive to preserve the function of malebenefit genes on the $\mathrm{Y}$ chromosome, and to acquire novel male-benefit genes on the Y [1-7]. These opposing effects lead to a heterogeneous structure of Y chromosomal DNA, with functional genes (often male specific, sometimes highly amplified) set among a sea of degenerate pseudogenes, repetitive sequence, and parasitic transposable elements.

The long arm of the mouse $\mathrm{Y}$ chromosome is a spectacular example of this process, being highly repetitive, transcriptionally silent in the majority of cell types, and yet indispensable for normal spermatogenesis [8-14]. Deletions on mouse Yq lead to teratozoospermia and reduced fertility. The severity of the phenotypes varies according to the 
extent of the deletion, with large deletions $(>=9 / 10$ of Yq) resulting in complete infertility $[8,13]$, while smaller deletions ( $\sim 2 / 3$ of Yq) result in reduced fertility and a less severe sperm shape abnormality $[9,11,14]$. Intriguingly, the offspring of males with $2 / 3 \mathrm{Yq}$ deletions show an approximately 60:40 sex ratio skew in favour of females [9], and this is due to reduced efficiency of Y-bearing sperm [15].

Recently, we have made considerable progress in defining the gene content of mouse Yq, identifying two new repeat gene families (Sly, Asty)[16] in addition to the one family previously known $(S s t y 1 / 2)$. During this work, we observed novel "recombinant" transcripts arising from loci that contain exons from both Ssty1 and Asty, and termed this new transcript Asty(rec) [16]. Here, we describe the detailed genomic arrangement of this rearranged locus and show expression of a large variety of transcriptional variants arising from these rearranged loci. These variant transcripts are differentially regulated during testis development.

We were also interested to know how these rearranged loci arose, and whether there were further examples of such "exon shuffling" on mouse Yq. We therefore compared the genomic organisation of the loci encoding all known $\mathrm{Yq}$ genes to each other and to their X-linked homologues, in order to more clearly delineate the composition of the novel rearranged loci, the differences between each of the Yq genes and their X-linked relatives, and the sequence of events involved in the genesis and amplification of these genes.

Finally, we investigated the wider genomic context of the rearranged loci by in silico mapping of the location of all known MSYq genes within the currently-released draft Y chromosome sequence contigs. The MSYq gene copies located by the mapping project were used to construct phylogenetic trees elucidating the sequence of events in MSYq evolution

\section{Results \\ A rearranged locus formed by chimerism between three Yq-specific genes}

While we previously reported the presence of both Ssty1 and Asty exons within the locus encoding Asty(rec) [16], further analysis of public genome sequence data shows this locus to be entirely composed of regions with high sequence similarity (greater than 90\% across all regions) to members of all three known Yq-linked gene families. The structure of this novel locus is shown in Figure 1A. It comprises sequence homologous to exons 1, 2 and partial exon 3 of Ssty1, followed by exons 2-4 of Asty, followed by exons 1-2 (and partial intron 2) of Sly in the opposite orientation. Since the locus contains segments of all three
Yq-linked gene families, we feel the designation Asty(rec) is no longer appropriate. Given its highly unusual composition, and the fact that it is transcribed in both directions, we propose the name Orly (Oppositely-transcribed, rearranged locus on the $\underline{\mathrm{Y}}$ ).

As previously detailed [16], a search of the nr database revealed two full-length cDNAs arising from Orly, both originating from the relict Ssty 1 promoter. In this article we will refer to these transcripts as Orly_v1 (accession number [GenBank:AK015935]) and Orly_v2 ([Genbank:AK016790], referred to in our previous work as Asty(rec)). The splicing patterns of Orly_v1 and Orly_v2 are shown in Figure 1B.

\section{Orly generates a wide diversity of alternative splice variants}

The existence of the Orly_v1 and Orly_v2 transcripts indicates differential splicing of the central exons in Orly. We performed a screen using primers from the various exons present in Orly_v2 (locations indicated in Figure 1C, sequences in Table 1) in order to see what further Orly splice variants were expressed. All primer pairs that gave an RT-PCR product from adult testis were tested using a range of normal mouse testis RNAs from different ages post partum in order to resolve the developmental onset of expression for each splice variant form (Figure 2). Primer pairs S1.f2/N2.r2 and N1.f/N2.r2 were also tested against a range of tissues to check tissue specificity of expression, and proved to be testis specific (Figure 3 ).

When using primer pairs directed at the outermost exons (S1 and N2), a single major band corresponding to Orly_v1 is observed, suggesting that this is the most abundant Orly transcript. Two faint larger bands were also detected by the S1.f2/N2.r2 primer pair (arrowed in Figure 3), however, we were unable to obtain sequence for these products. These upper bands are likely to represent transcripts including portions of Ssty1 exons 2/3 or Asty exons 2-4, as detected in the other reactions (see below). No larger bands were seen in the S1.f1/N2.r1 reaction. It is possible that the larger bands detected by the S1.f2/ N2.r2 primer pair arises from copies of the Orly locus where the exon S1.f1 and/or N2.r1 primer binding sites are mutated.

Using primer pairs directed at the other Orly_v2 exons gave a wide variety of bands. Many of the products generated from RT-PCR on adult testis were gel purified and sequenced to confirm which regions of Orly are included in each detected transcript. Unfortunately we were unable to generate clean sequence for the products produced by the S3a.f/A3.r primer pair. This is likely due to the presence of a large number of similarly-sized transcripts which cannot be separated on a gel. The resulting partial tran- 


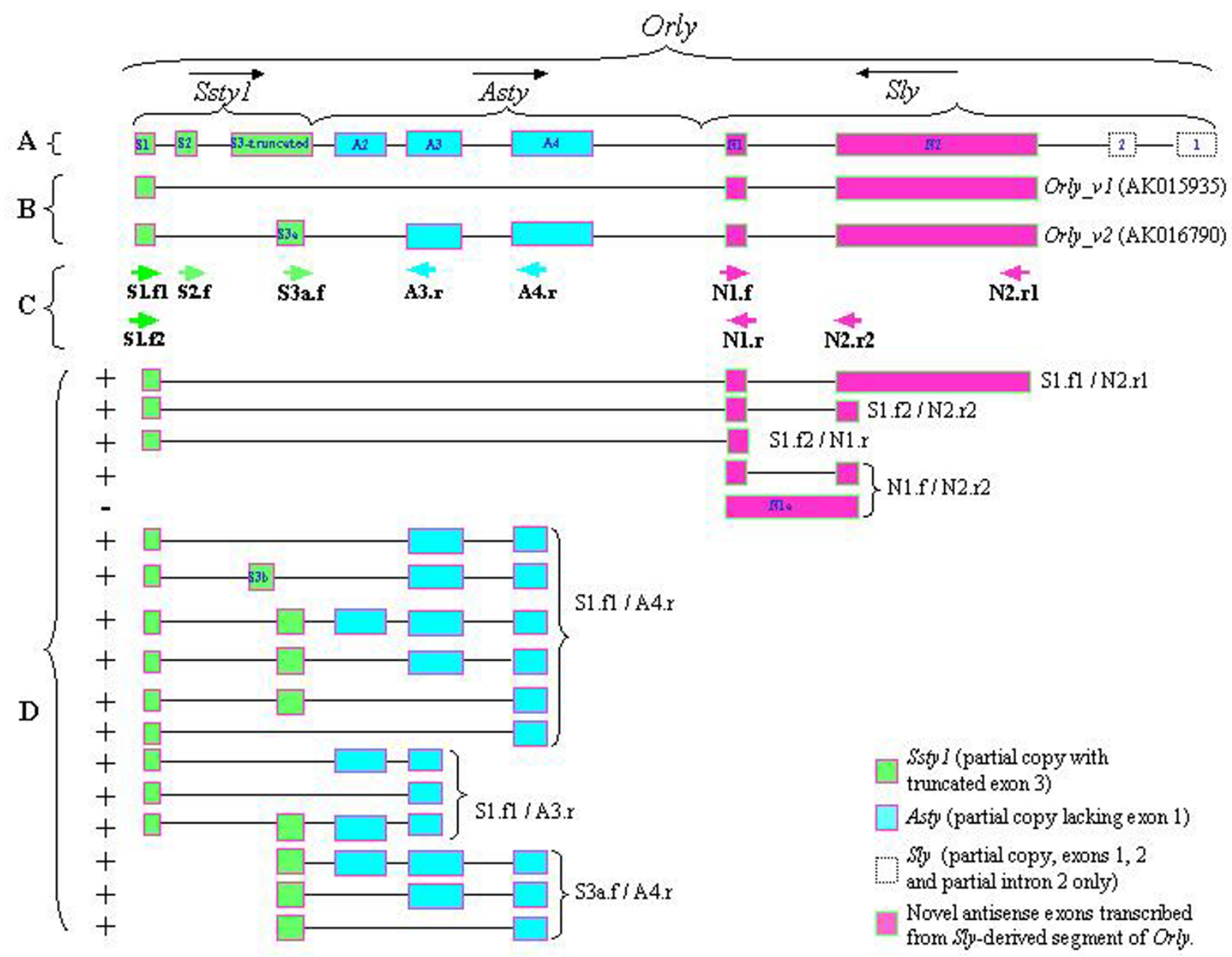

Figure I

Characterisation of Orly transcripts. A: Structure of Orly as a chimera of relict partial Sstyl, Asty and Sly loci (not to scale). Arrows indicate orientation of the relict loci. Exons are coloured according to their origin. B: Structure of Orly transcripts found in the $\mathrm{nr}$ database. C: Primer locations used to characterise Orly splice variants (sequences given in Table I) D: Structure of variant transcripts detected in the screen. The primer pairs which amplify each variant form are indicated. Exons $3 a$ and $3 \mathrm{~b}$ are short forms of Sstyl-derived exon S3, and are variably included in Orly transcripts. Of note is that the majority of splice variant forms do not conform to the splicing patterns of the known transcripts shown in panel A. + indicates "forward" transcripts - indicates the "reverse" transcript detected with primer pair NI.f/N2.r2

scriptional map for Orly is shown in Figure 1D. In most cases, the sequenced bands correspond to spliced transcripts, however, the majority of the products do not conform to the splicing pattern of Orly_v1 or Orly_v2. It appears that there is a plethora of different Orly isoforms expressed at low levels, which are only detected when specific primers are used.

We investigated whether any of the detected Orly isoforms had any significant coding potential. The Ssty 1 open reading frame is encoded by exon 3 of $S s t y 1$, which is not fully included in any Orly transcriptional variant (though two shorter forms of this exon are variably included). Orly transcripts thus do not encode the SSTY1 protein. The Sly portion of the locus is in antisense, thus Orly transcripts cannot encode any portion of SLY. Finally, Asty does not contain any open reading frame, thus the Asty-related portion of Orly also has no coding potential. Further electronic searching of the various Orly transcriptional variants revealed no significant open reading frames other than a partial degenerate retroviral pol sequence (see below). 
$\begin{array}{llllll}19 & 21 & 23 & 29 & 35 & \text { A }\end{array}$
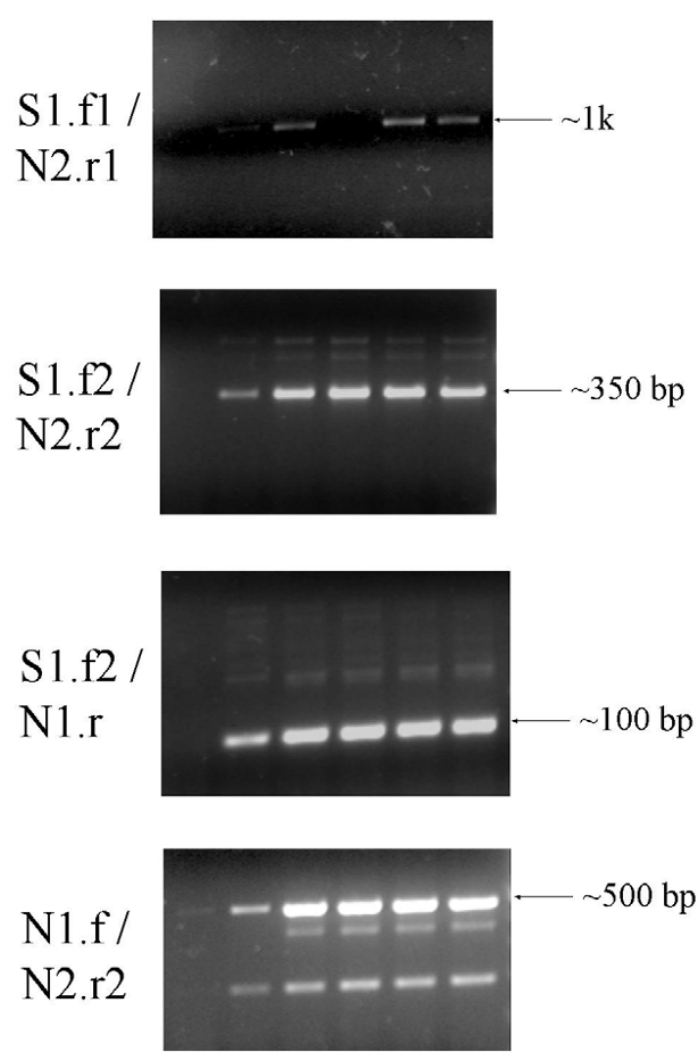

$\begin{array}{llllll}19 & 21 & 23 & 29 & 35 & \text { A }\end{array}$

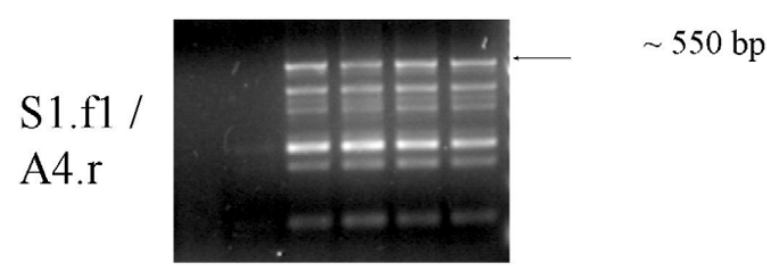

S1.f1 /

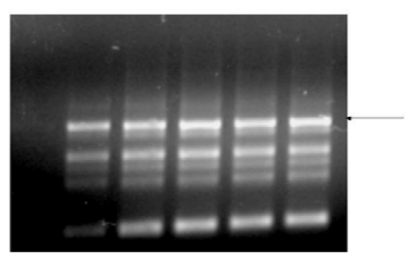

$\sim 450 \mathrm{bp}$

A3.r

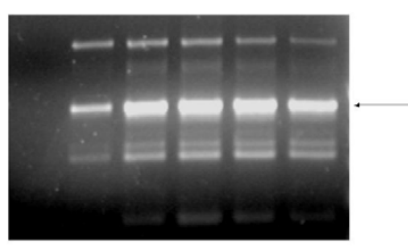

$\sim 450 \mathrm{bp}$

S3a.f/

A4.r

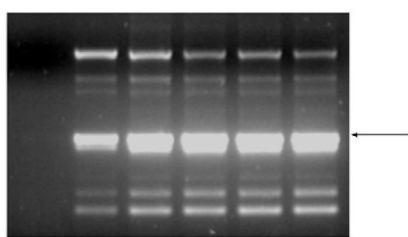

$\sim 350$ bp

Figure 2

RT-PCR reactions demonstrating the wide range of Orly splice variants expressed in developing testis. RT-PCRs were performed on RNA from testes at 19, 21, 23, 29 and 35 days post partum, and on adult ( 8 week) testes. Size is indicated for one band in each case.

\section{Orly retains potential promoter sequence from both Ssty I and Sly}

The regions of high sequence identity between Orly and its various progenitor loci extend a further $5 \mathrm{~kb}$ into the upstream region of Ssty, and $3 \mathrm{~kb}$ into the upstream region of $S l y$, indicating that the rearranged locus has retained the proximal upstream promoter regions of both genes, in antisense orientation relative to each other (Additional File 1). Orly_v1 and Orly_v2 are known to be transcribed from the relict Ssty1 promoter [16], which is thus shown to be functional.

Turning to the relict Sly promoter region, we conducted a search for transcription factor binding sites using TFSEARCH [17]. This showed that of the 42 predicted transcription factor binding located between -600 and +10 of the reference Sly sequence, 35 were present at the corresponding site in Orly, indicating retention of potentially functional promoter elements (Additional File 2). Overall sequence identity between Sly and Orly across this region is $95.7 \%$. Significantly, the conserved elements include a GCCAAT box at position -161 of the reference Sly locus. This motif is a strong transcriptional signal, and is known to be present in other spermatid specific TATAless promoters such as the Pgk-2 promoter [18].

\section{Both of the promoters at opposite ends of Orly are functional}

This electronic promoter analysis suggested to us that the relict Sly promoter at the 3' end of the Orly locus may have retained functionality, and be able to generate oppositestrand transcripts. We designate such opposite-strand transcripts as Orlyos. No Orlyos transcripts were present in the nr or dbEST databases. We used strand-specific RTPCR to determine whether any of the bands shown in Figure 2 corresponded to Orlyos transcripts.

Importantly, of the three bands obtained using the N1.f/ N2.r2 primer pair, the lower band corresponds to a forward orientation (Orly) transcript, while the upper band 

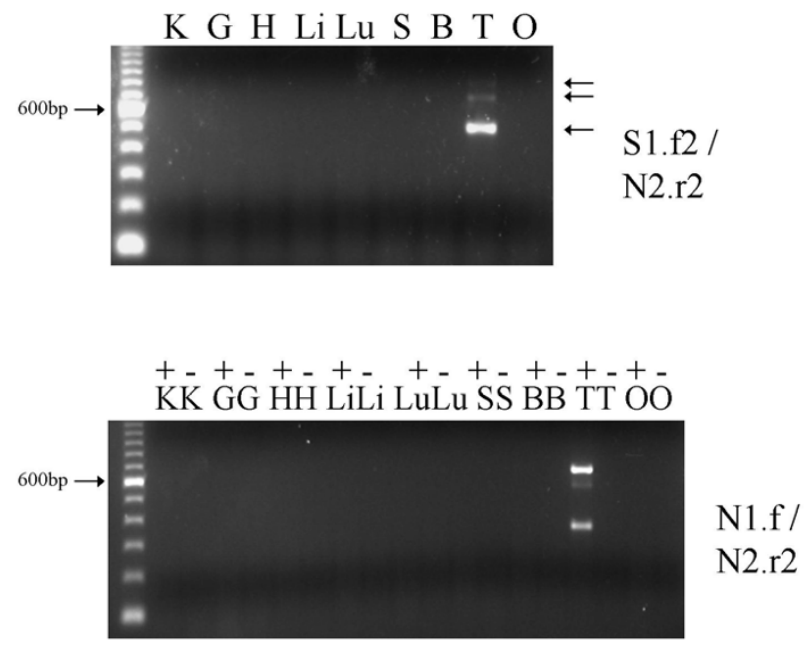

\section{Figure 3}

RT-PCR using primer pairs SI.f2/N2.r2 and NI.f/N2.r2 across a range of different tissues. $\mathrm{K}=$ kidney, $\mathrm{G}=$ gut, $\mathrm{H}=$ heart, $\mathrm{Li}=$ liver, $\mathrm{Lu}=$ lung, $\mathrm{S}=$ spleen, $\mathrm{B}=$ brain, $\mathrm{T}=$ testis, $\mathrm{O}$ = ovary. For the NI.f/N2.r2 primer pair, reactions were performed with (+) and without (-) reverse transcriptase. The absence of a band in the - controls demonstrates that the larger unspliced products are true transcripts and not due to DNA contamination.

corresponds to a reverse orientation transcript (Figure 4). Sequencing confirmed that this RT-PCR product shows 99\% identity to the reference Orly genomic sequence over $622 \mathrm{nt}$, and only $91 \%$ identity to Sly over $464 \mathrm{nt}$. This demonstrates that this transcript arises from an Orly locus rather than from Sly, and constitutes a true Orlyos transcript. The middle band seen in this reaction is not always detected by RT-PCR, and we were unable to determine the direction of transcription of this band.

Of the other splice variants shown in Figure 2, all were confirmed by strand-specific RT-PCR to be "forward"

Table I: Primers used to characterise Orly transcripts (locations shown in Figure IC)

\begin{tabular}{|c|c|}
\hline Primer & Sequence \\
\hline SI.fI & CCCAACGTTGTTCCTCAAGT \\
\hline SI.f2 & TCCCAGTGGTGTATGAAAGG \\
\hline S2.f & CGGAGTTGCAGTGGCAAATA \\
\hline S3a.f & AGCTCCTGGATGACTACAAG \\
\hline A3.r & GCAACTCCTCATCAGAGACT \\
\hline A4.r & CGATGATGAGTGACCTAAAGAT \\
\hline NI.f & CAAGATTTGGCATCTGTAAGG \\
\hline NI.r & GCCATTGTCTGATGAAAGTACC \\
\hline N2.r2 & CCAGAAGCGGTTACATGC \\
\hline N2.rI & AGCTACCTGAGGAATTGAGAT \\
\hline
\end{tabular}

(Orly) transcripts (data not shown). This is unsurprising as the primers were designed against the forward transcript Orly_v2. Orlyos transcripts must necessarily have different exon boundaries, which presumably do not include the majority of the primer locations included in our screen.

\section{The terminal exons of Orly derive from a retrovirus and are in antisense to Sly and Orlyos}

Both of the known Orly transcripts terminate with two novel exons with antisense homology to intron 2 of $S l y$ (see Figure 1A, exons N1 and N2). As discussed above, this section of Orly is also transcribed in the opposite orientation, generating Orlyos transcripts. Thus there is the potential for Orly transcripts to form dsRNA either by pairing with Orlyos transcripts or with nascent Sly transcripts.

Exons $\mathrm{N} 1$ and $\mathrm{N} 2$ derive from a partial degenerate retrovirus belonging to the MuRVY lineage of mouse $\mathrm{Y}$ chromosome specific repeats [19], which is embedded in this intron of Sly (see below). Orly-F transcripts terminate at the transcription stop site of this MuRVY-related element. We therefore deduce that although the MuRVY element is degenerate and does not encode a functional retrovirus, its transcriptional termination site has remained functional and become co-opted to form the transcriptional termination site for Orly forward transcripts. None of the known Orly transcripts contain any large open reading frames, however, Orly_v1 contains a short ORF running from bases $117-284$. This ORF has $63 \%$ identity and 75\% similarity over 49 aa to a partial pol gene (data not shown), further demonstrating the retroviral origin of the terminal exons of Orly.

\section{Tissue- and developmental stage-specific expression of Orly isoforms}

Orly transcripts are under tight transcriptional control. All variant forms (both forward and reverse) are only observed after day 19 of postnatal life, and thus are deduced to be spermatid-specific. This is to be expected as both Ssty1 and Sly promoters are spermatid specific $[9,13,16]$. The age of first appearance for each band varied from day 19 to 23, indicating differential regulation of Orly isoforms in successive spermatid stages. This variation was observed both between different primer pairs (e.g. the majority of S1.f1/A4.r bands appear at $23 \mathrm{dpp}$, while the majority of S1.f1/A3.r bands appear at $21 \mathrm{dpp}$ ), and between different bands detected by the same primer pair (e.g. the upper, lower and middle bands in the N1.f/ N2.r2 reaction appear at 19, 21 and 23 days respectively). This differential regulation may be due to spermatid stage dependent splicing of transcripts, or may represent varying subsets of transcripts arising from different copies of Orly with subtly different promoter activities. It is unfortunately not possible to use in situ or Northern blot data 


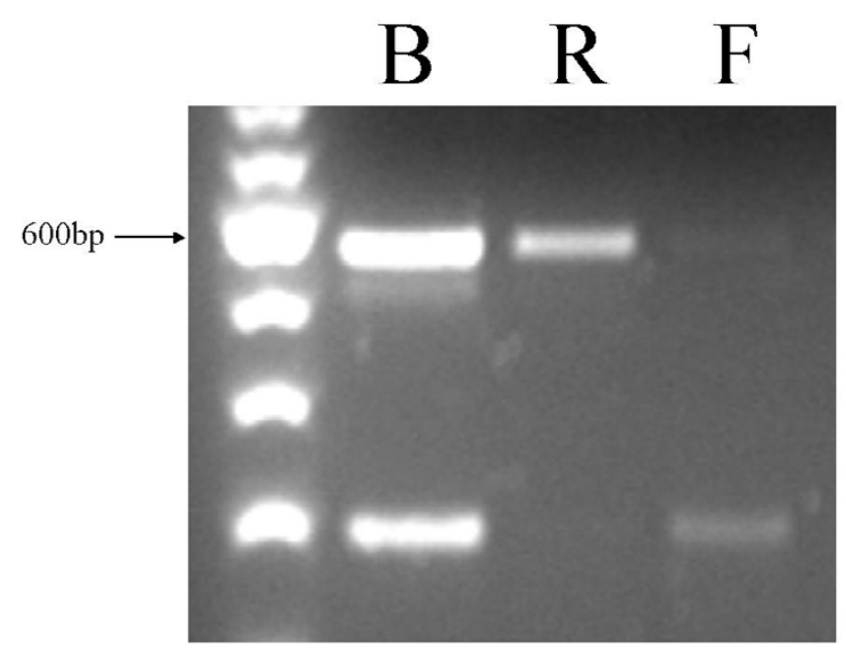

Figure 4

RT-PCR on adult testis using primer pair NI.f/N2.r2. B: Both primers present during RT step, both products are detected. R: RT reaction was primed with NI.f and detects a "reverse" transcript. F: RT reaction was primed with N2.r2 and detects a "forward" transcript.

to confirm the detailed cellular expression patterns of these transcripts, since there is no portion of any of them which is not also part of a different Y-linked gene or retrovirus with a confounding expression pattern.

\section{Genomic comparisons of Orly, its progenitor loci, and their $X$ homologues}

We carried out a detailed comparison of the genomic loci encoding Orly, the other MSYq genes and their X counterparts, in order to better delineate the sequence of events during MSYq evolution.

\section{Genomic comparison of Sstyl and Ssty 2}

The structure of the reference genomic loci encoding Ssty $1 / 2$ is shown in Figure 5 (see Additional File 3 for ClustalW alignment). Sequence identity is $82.7 \%$ across the locus as a whole. The coding region is conserved (barring the final 5 amino acids of Ssty1), and lies entirely within exon 3. Sequence identity within the coding region is $86.4 \%$. The splice site at the end of the first exon is conserved. Ssty2 does not incorporate sequence corresponding to exon 2 of Ssty1. The splice site at the start of the terminal exon is located differently in Ssty1 and Ssty2, the latter thus having a longer terminal exon which in part matches intronic sequence from Ssty1. The 5'UTRs of the two gene transcripts are thus very different despite the high sequence identity between the loci. An X-linked member of this gene family (Sstx) is known, however, the similarity at the nucleotide level is low except for a small segment at the start of the coding region (76\% over 135 bp) [Paul Burgoyne, personal communication].

\section{Genomic comparison of Xmr and XIr}

The structure of the reference genomic loci for Xmr and Xlr is shown in Figure 6. Xmr has two transcriptional variants arising from alternative transcription start sites, as indicated in this figure. The structure of $X l r$ is similar to the shorter transcriptional variant of $X m r$, however, there is considerable sequence divergence in exon 4 of $X l r$. In particular, the $3^{\prime}$ portion of Xlr exon 5 does not match any $\mathrm{Xmr}$ sequence. The longer transcript derived from the Xmr locus contains 4 additional 5 ' exons which do not show sequence similarity to the upstream region of the $X l r$ locus. These exons thus represent either novel sequence acquired by $X m r$ following $X m r / X l r$ divergence, or deletion of the 5 ' portion of the Xlr locus.

There are two partial degenerate LINE elements within the $\mathrm{Xmr}$ locus, the first lying in the second intron of the longer isoform, and the second lying in the sixth intron (and thus also present in the fourth intron of $X l r$ ). In addition to these degenerate LINEs, Xmr also contains a full-length LINE element from the L1MD-A2 lineage, which includes upstream monomer repeats and thus is potentially transcriptionally active [20]. The element lies in intron 7 of $\mathrm{Xmr}$ but is not found in the corresponding location (intron 6) of $X l r$, indicating that the LINE insertion occurred subsequent to Xmr/Xlr divergence.

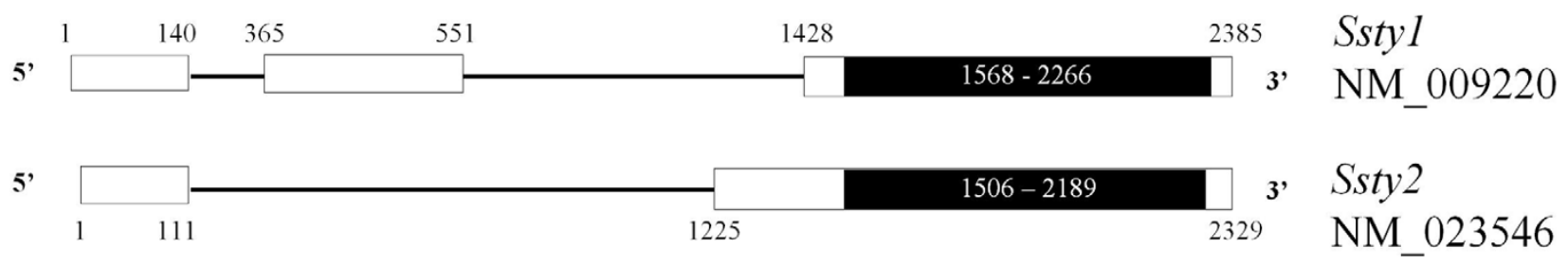

\section{Figure 5}

Genomic arrangement of Ssty //2. Numbers indicate nucleotide position within the genomic locus. White boxes indicate exons, with the coding region shown in black. 


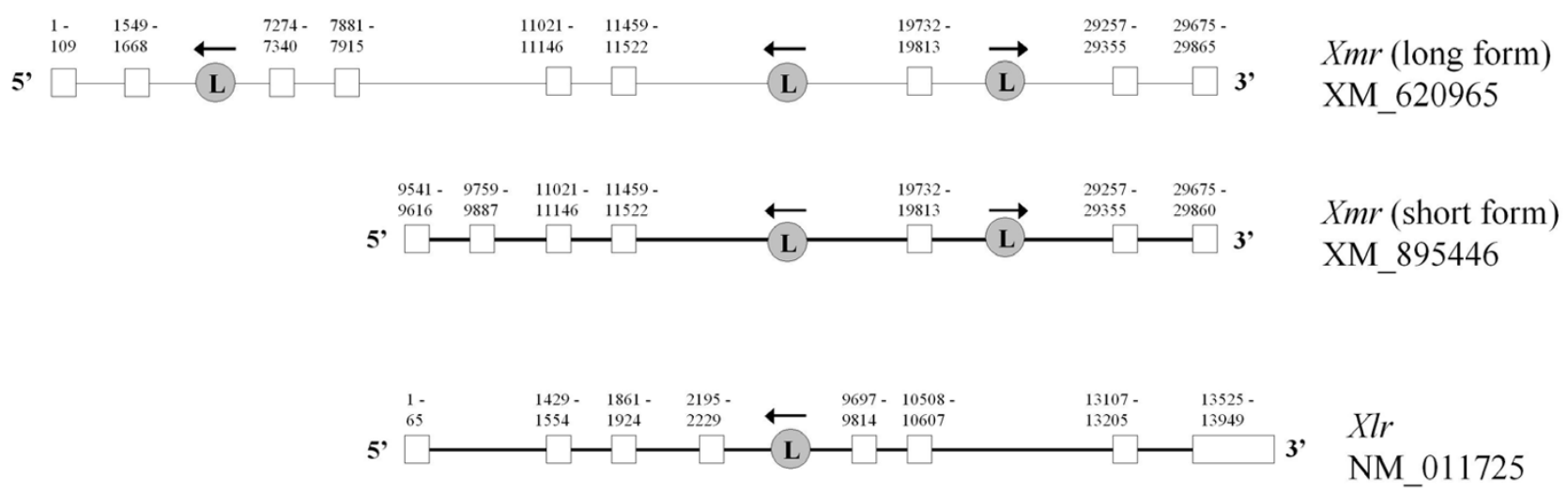

Figure 6

Genomic arrangement of Xmr and XIr. L indicates the position and orientation of LINE elements. Xmr contains a LINE insertion in intron 7 that is not present in Xlr and presumably postdates $\mathrm{Xmr} / \mathrm{XIr}$ divergence. There are two transcriptional variants of Xmr, neither of which splice in an exon corresponding to XIr exon 5. This exon encodes the nuclear localisation signal, thus $\mathrm{Xmr}$ has lost the ancestral nuclear pattern of expression. The longer Xmr isoforms has 4 unique $5^{\prime}$ exons.

Sly arose as a chimeric gene via fusion of Xmr and XIr Sly, the Yq-linked member of the family, is a chimeric gene formed by fusion of the 5' portion of Xmr to the 3' portion of Xlr. Figure 7 shows the structure of Sly together with the homologous regions of Xmr and Xlr.

The origin of the 5 ' end of Sly is demonstrated by exons $1-4$, which match the 4 additional exons uniquely present in the longer Xmr isoform. The origin of the 3' end of Sly is demonstrated by exons $7-10$, which match the final exons of Xlr including the Xlr-specific portion of exon 5. Sly lacks the L1MD-A2 element present in Xmr intron 7, further confirming the chimeric nature of this gene as an $\mathrm{Xmr} / \mathrm{Xlr}$ hybrid. Exons 5-6 of Sly arose via duplication of exons 3-4 and show 88/102 nucleotide identity to these exons. There are degenerate LINE elements at the borders
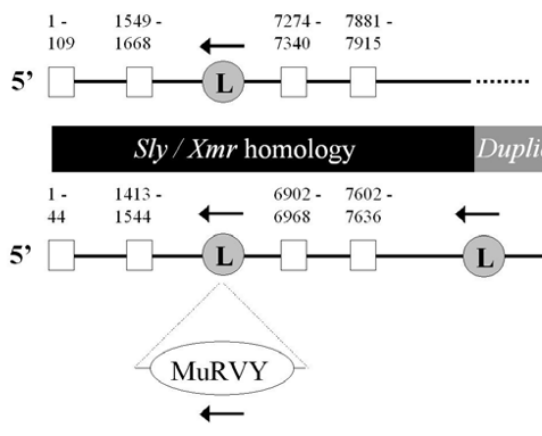

Xmr exons 1-4

XM_620965

Sly

NM 201530 Duplication of Xmr exons 3-4 $\begin{array}{lll}7602- & 14661- & 153 \\ 7636 & 14727 & 153\end{array}$ $22487-23388-$ $25619-25199$ $\square$ - $\square$,

\section{L $-\square$ \\ Sly/Xlr homology}

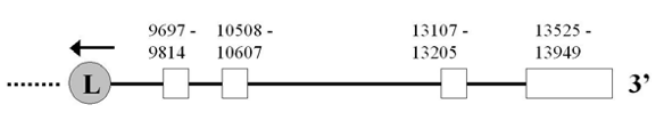

Xlr exons 5-8

NM_011725

\section{Figure 7}

Genomic arrangement of Xmr, XIr and Sly. Sly exons I-4 are homologous to Xmr exons I-4. Sly exons 5-6 represent an internal duplication of exons 3-4. Sly exons 7-10 are homologous to Xlr exons 5-8. There are LINE elements at the borders of

each section, suggesting that Sly was formed by recombination between LINE repeats.

(L) indicates the location and orientation of these LINE elements. 
of this duplication event, and also at the border between the Xmr-derived and Xlr-derived segments of Sly, thus it is likely that recombination between LINE elements was responsible for the creation of Sly.

The LINE element present in intron 2 of Sly is interrupted by a stretch of DNA with distant sequence similarity to the mouse MSYq-specific retrovirus, MuRVY. This LINE element is uninterrupted in the progenitor Xmr, thus we conclude that the MuRVY insertion occurred subsequent to the creation of Sly. The MuRVY-related sequence is inserted in antisense orientation relative to Sly itself. The extent of the MuRVY-related stretch of DNA varies between Sly copies (see phylogenetic tree analysis below), but in all cases the terminal portion (including MuRVY transcription termination site) is retained. RepeatMasker analysis [21] of the insert shows 13.1\% divergence, $13.1 \%$ deletion and $3.2 \%$ insertion relative to the consensus MuRVY LTR sequence, and 32.5\% divergence for non-LTR portions of the insert. As discussed above, the MuRVYrelated sequence in Sly intron 2 forms the source for the terminal Orly exons.

Recent work has shown that both Xmr encodes a cytoplasmic protein, in contrast to the protein encoded by $X l r$, which is nuclear[22]. The KRKR nuclear localisation signal in $X l r$, which is conserved from the autosomal progenitor gene SCP3, is located in exon 5. Xmr does not include this exon, suggesting that this is the reason for the altered protein localisation. Interestingly, this signal is mutated to KRKW in the corresponding portion of Sly.

\section{Genomic comparison of Asty/Astx}

As reported previously [16], Asty and Astx have an identical genomic organisation, and share 95\% sequence identity across introns and exons.

\section{The genomic context of Orly}

We used BLAST comparison to search for all copies of each Yq-linked gene (Ssty1/2, Asty, Sly, Orly) in the currentlyreleased draft sequence contigs [Mouse Chromosome Y Mapping Project (Jessica E. Alfoldi, Helen Skaletsky, Steve Rozen, and David C. Page at the Whitehead Institute for Biomedical Research, Cambridge MA, and the Washington University Genome Sequencing Center, St. Louis $\mathrm{MO})]$.

We then used this information to generate a "fingerprint" for each available Yq contig, noting the order and orientation of the various copies of each gene present in each contig (see Additional File 4). Interestingly, we found that Orly always has the same genomic context, being flanked downstream by Ssty1 and upstream by Ssty2, with both loci in the same orientation as Orly. The neighbouring copies of Ssty1 all contain a SINE insertion at position
393, and form a distinct sub-group within the phylogenetic tree (see below: bootstrap support value for this clade is $1000 / 1000$ replicates).

Using the fingerprints as a guide, we were able to assemble a "super-contig" containing 3 copies of Ssty1, 3 copies of Ssty2, two copies of Asty, two copies of Sly and one copy of Orly. In all, 13 of the $33 \mathrm{Yq}$ contigs are congruent with this super-contig ordering, and a further 4 contigs appear to be slight variants upon it. This "super-contig" indicates the presence on mouse Yq of a highly amplified repeat unit of greater than $500 \mathrm{~kb}$ in length, which presumably corresponds to the Huge Repeat Array reported at conferences by Alfoldi et al [23]. Sequence identity between the various contigs contributing to this "super-contig" is very high (>98\% excluding indels), indicating substantial homogeneity between copies of the Huge Repeat.

Figure 8 shows the layout of the Huge Repeat unit, and the contigs that match this ordering. Figure 9 is an example Dotter alignment of two Huge Repeat contigs, [GenBank:NT 161892] and [GenBank:NT 161926], demonstrating that high sequence homology extends across the entire contig, genic and intergenic regions included. In all, these two contigs share $98.26 \%$ nucleotide identity over 235813 nt. The Huge Repeat is itself internally repetitive it can be seen in Figure 8 that there is a repeating segment containing Ssty1, Asty and Sly in order. Figure 10 is a Dotter plot comparing [GenBank:NT_165794] to itself, demonstrating this internal repeat. The repeat region in this contig shows $98.12 \%$ identity over 179584 nt.

However, many contigs did not fit the Huge Repeat consensus ordering. Two further classes of Yq contig were identified (see Table 2): Ssty/Asty-enriched contigs $(n=7)$ and Sly /Asty-enriched contigs $(\mathrm{n}=5)$, while 4 contigs remained unclassifiable. These non Huge Repeat contigs were also highly internally repetitive. Figure 11 is an example Dotter plot comparing [GenBank:NT_161904] to itself. The structure is of a tandem repeat unit of $\sim 120$ $\mathrm{kb}$ embedded inside a larger tandem repeat of $\sim 210 \mathrm{~kb}$. In all, the repeated segment in NT_161904 shows $97.78 \%$ identity over $204851 \mathrm{nt}$.

\section{Dynamics of Yq gene family expansion}

A key question is whether these four gene families (Ssty1) 2, Asty, Sly and Orly) were amplified separately on Yq during mouse evolution, or whether there was a single period of amplification increasing the copy number of all genes simultaneously.

We constructed a set of phylogenetic trees using the neighbour-joining method of Saitou \& Nei [24], comparing Ssty1-, Asty- and Sly-derived regions of Orly to the corresponding regions of the progenitor loci (Figures 12, 13, 


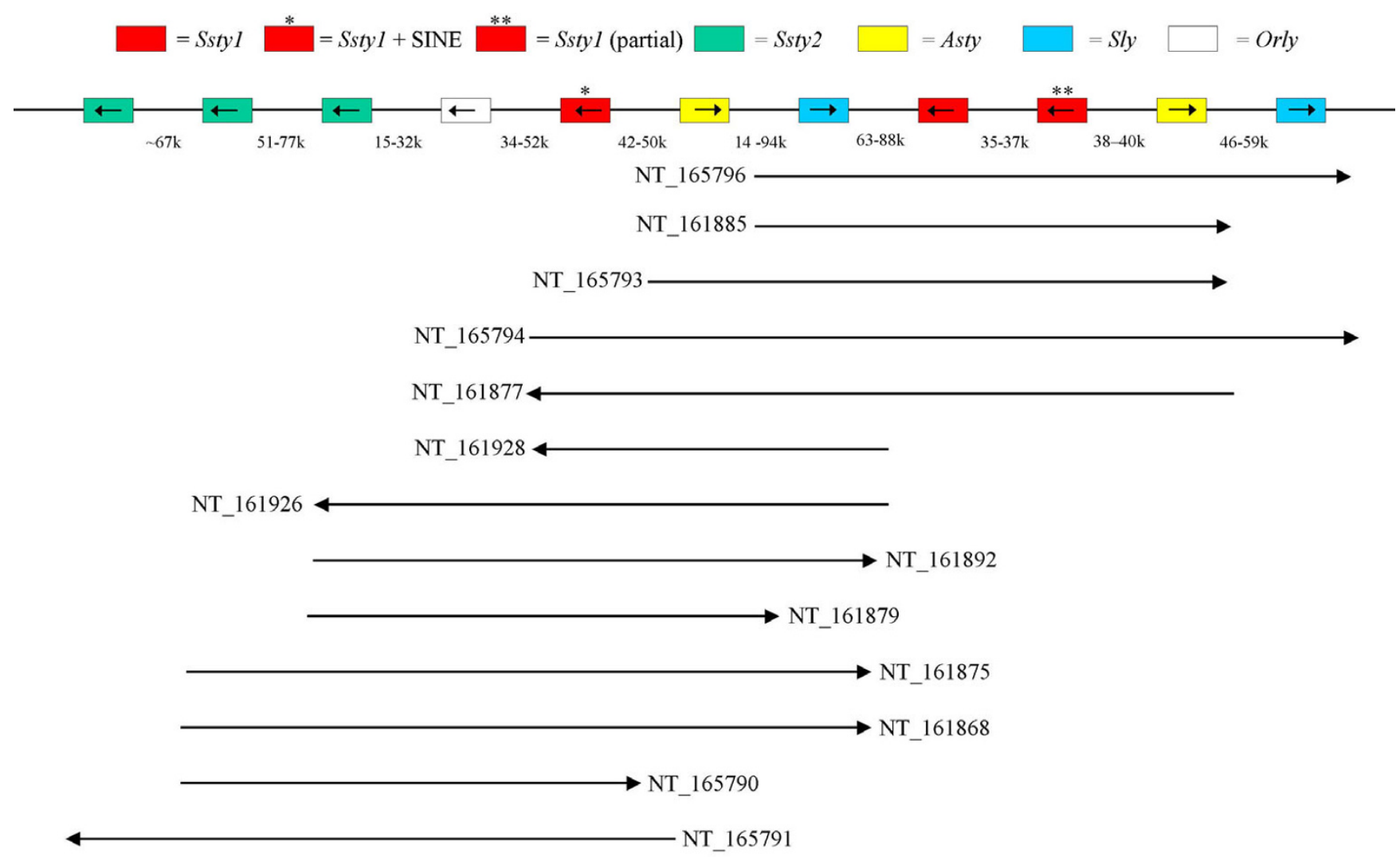

\section{Figure 8}

Schematic diagram showing the layout of the Huge Repeat Array repeat unit on mouse Yq. Order and orientation of Yq gene copies is shown, with individual contigs represented as line segments below. The size range of the interval between each gene locus is shown. The repeat unit is at least $500 \mathrm{~kb}$ in length, but our current analysis is unable to define the complete extent of the repeat. The Ssty I copies within the repeat can be distinguished from each other based on the presence or absence of a SINE insertion, and by a deletion removing exons I-2 of one copy of Sstyl (see also Figure I0).

14). For the Asty- and Sly-related trees, we were able to use the X-linked homologue as an outgroup to root the tree, however, for the Ssty-related tree this is not possible owing to much greater divergence of the $\mathrm{X}$ homologue which precludes accurate alignment. For this tree, the Ssty 2 clade was used as the outgroup to root the tree. Bootstrap analysis of 1000 replicates was used to establish the robustness of all three trees.

From this phylogenetic analysis we observe:

- In all three cases, Orly sequences form a discrete clade (bootstrap support value of 1000/1000 replicates for all three trees).

- Gene copies lying within the Huge Repeat contigs also form distinct clades in all three trees (bootstrap support of $960 / 100$ to $1000 / 1000$ in all cases). Note that each copy of the Huge Repeat unit contains several copies of Ssty1,
Ssty 2 and Asty. These three genes thus give rise to several Huge Repeat-associated clades in each tree. Each of these clades contains the gene copies from matching locations within the Huge Repeat unit.

- A final set of contigs forms a distinct clade in both the Ssty and Asty-related trees. This clade contains a group of Ssty1 /Asty-enriched contigs, [GenBank:NT_161904], GenBank:NT_161906, GenBank:NT_161911] (bootstrap values $969 / 1000$ to $1000 / 1000$ in the two trees). At slightly lower confidence levels, this clade also includes [GenBank:NT 165795] (bootstrap values 902/1000 to $989 / 1000$ in the two trees).

- At the time of Orly divergence, the Ssty family was already moderately amplified on the Y, with 8 Ssty1 lineages and 13 Ssty2 lineages present. By contrast, at the time of Orly divergence, there were only $\sim 4$ Asty lineages and 1 Sly lineage present on the $\mathrm{Y}$ 


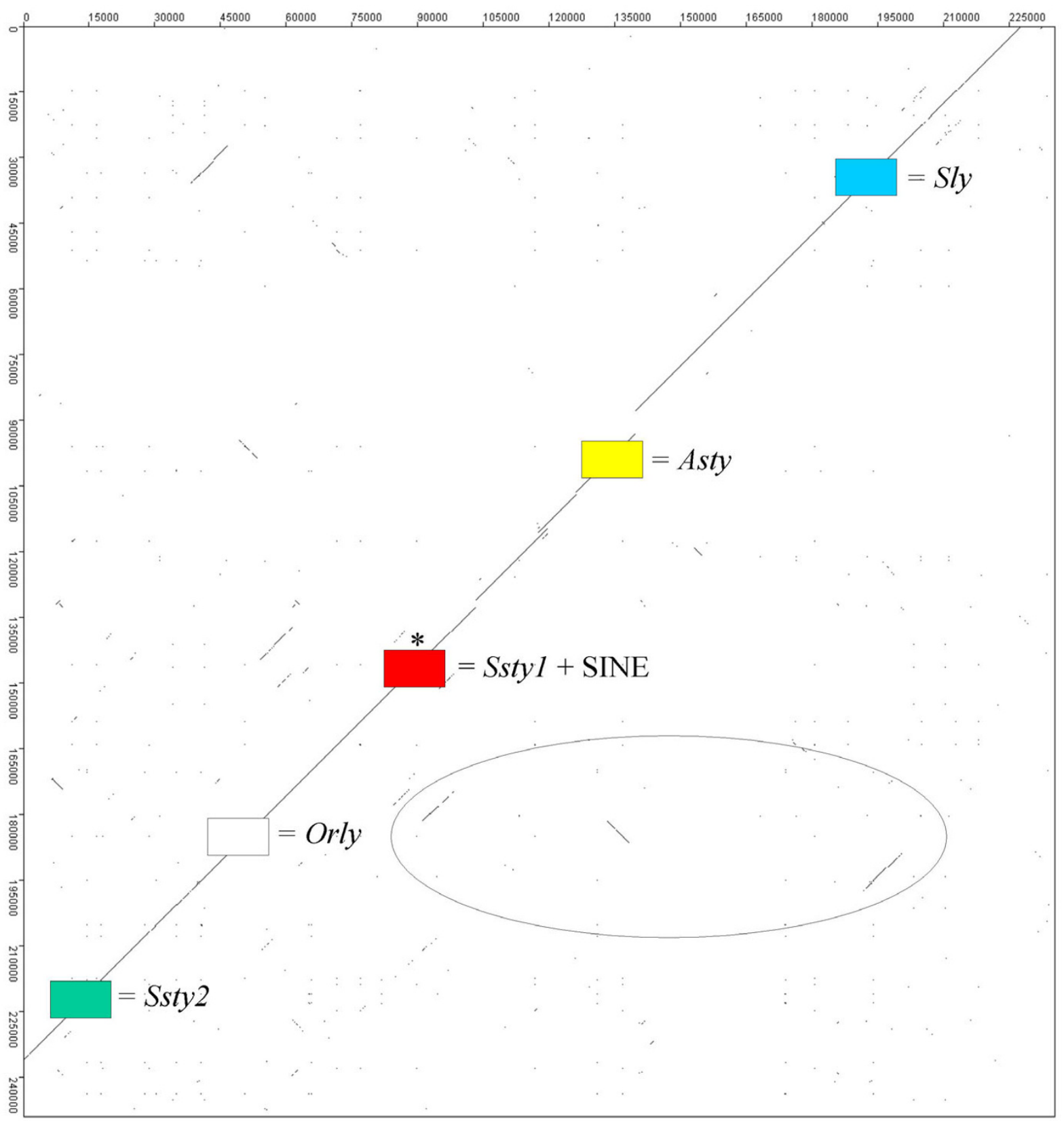

\section{Figure 9}

Dot plot comparing [GenBank:NT 161892] to [GenBank:NT 161926]. This demonstrates the very high degree of sequence identity between copies of the Huge Repeat. Locations of gene loci within the contigs are indicated. The homology between Orly and its component loci is clearly visible as three short internal repeat sections within the contigs (ringed).

- In all three cases, there was a massive amplification of gene copy number subsequent to Orly divergence. This amplification occurred predominantly in branches of the phylogenetic tree corresponding to Huge Repeat contigs, however, there was also amplification of a Ssty1 /Astyenriched clade subsequent to divergence of the Orly clade. 


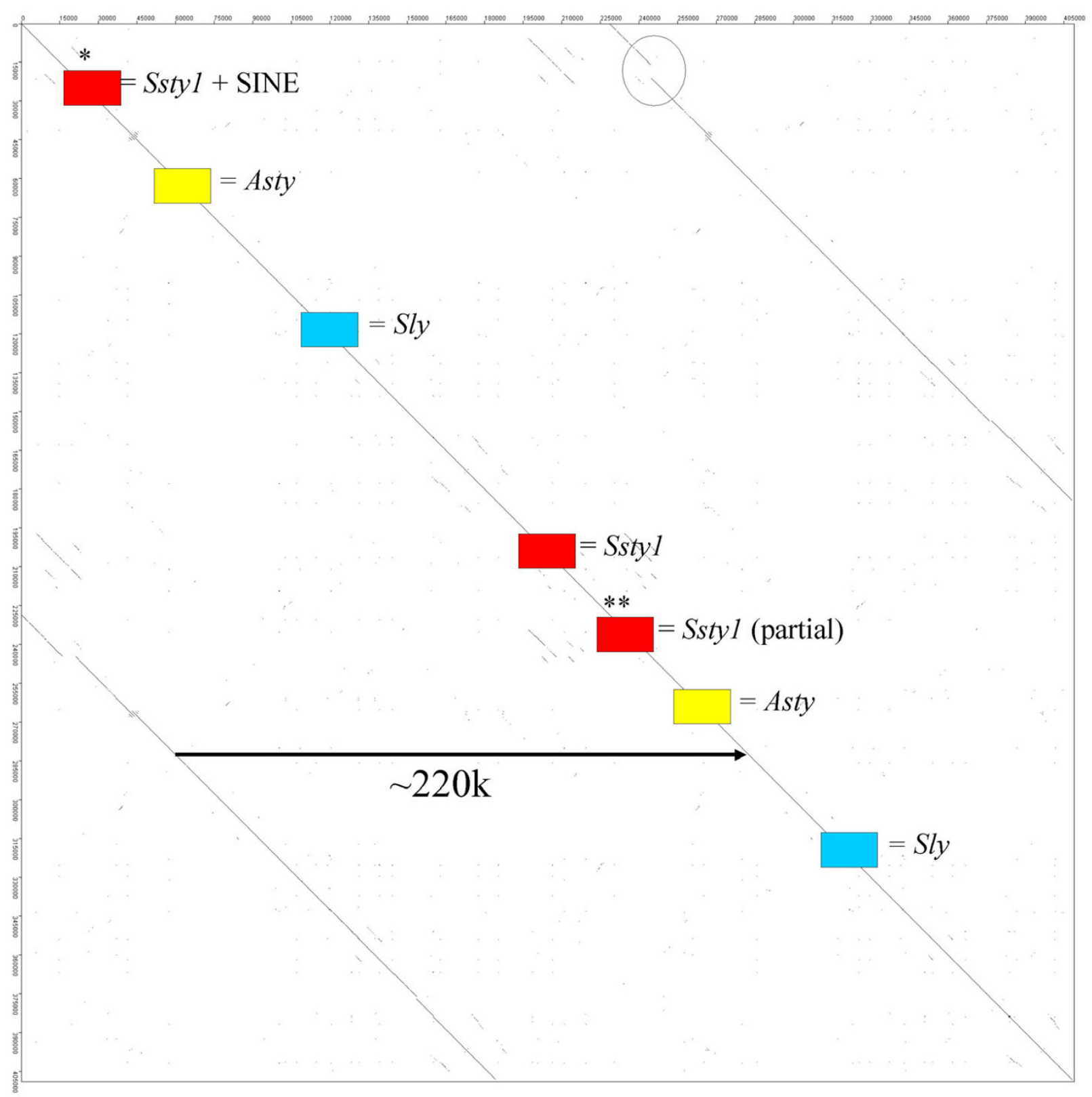

Figure 10

Dot plot comparing [GenBank:NT 165794] to itself, in order to demonstrate the nested repeat structure of the Huge Repeat Array. Although the repeat unit of the Huge Repeat Array spans at least $500 \mathrm{k}$ (see Figure 8), it also contains an internal repeat with a unit length of $\sim 220 \mathrm{k}$, containing copies of Ssty I, Asty and Sly. Visible (ringed) is a small deletion in one of the repeat units, which has deleted the first two exons of the Sstyl copy located in the region.

From these trees, we also observed that all genes within each family showed very similar degrees of divergence from the root of the tree in all cases. This is to be expected as all three trees were based on noncoding sequence. The sequence used to build the trees is thus likely to be evolving at nearly neutral rates. Given nearly neutral rates of evolution, the degree of sequence divergence forms a "molecular clock" indicating the timing of the various 


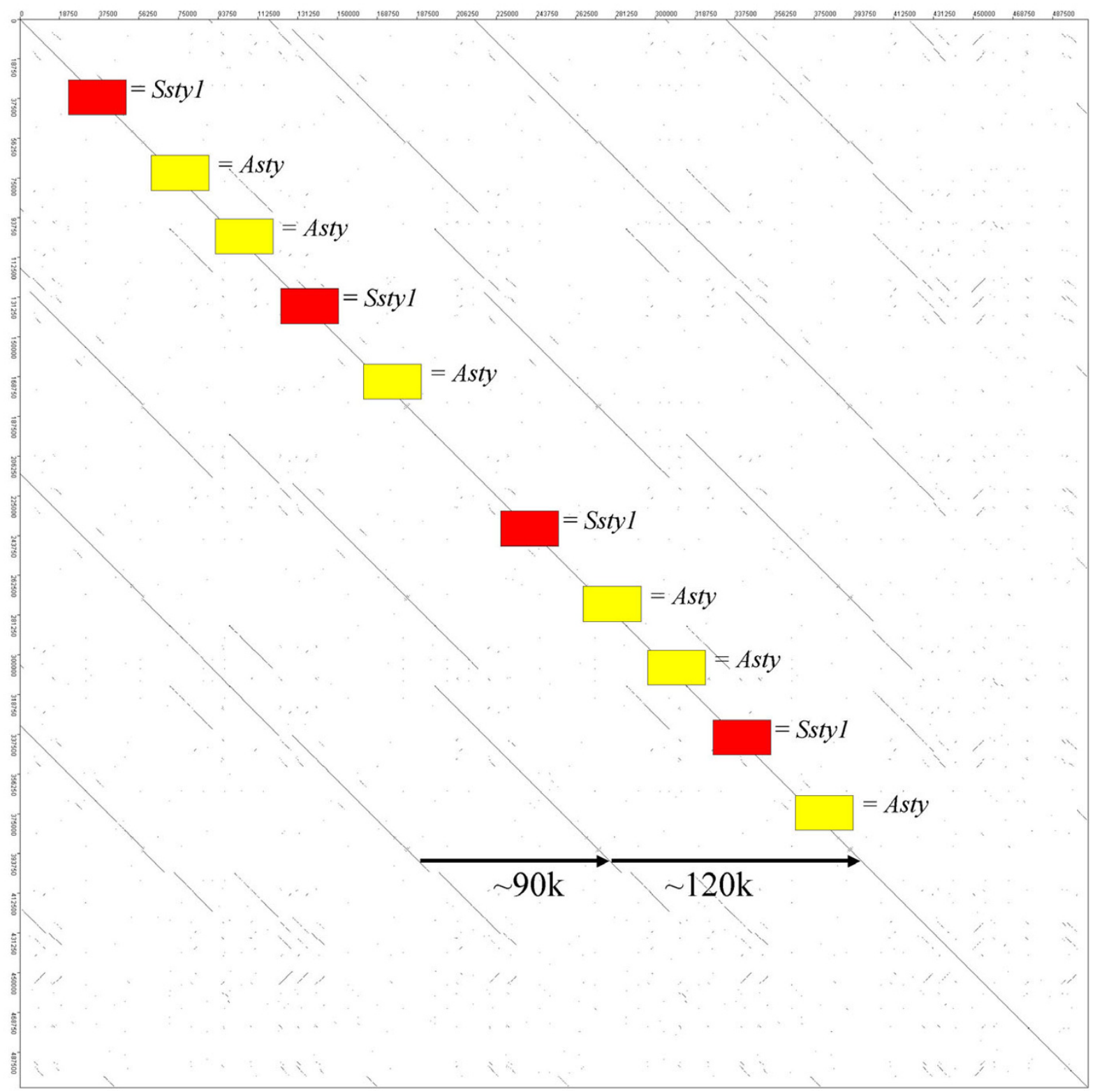

\section{Figure I I}

Dot plot comparing [GenBank:NT 161904] to itself. This contig is not part of the Huge Repeat, but forms part of an Ssty l/Asty enriched subset of $Y q$ contigs. This class of $Y q$ sequence also appears to have been amplified in recent mouse evolutionary history, concurrently with Huge Repeat Array expansion. This contig contains nested direct repeats of $\sim 90 \mathrm{k}$ and $\sim 120 \mathrm{k}$, giving a total repeat length of $\sim 210 \mathrm{k}$.

events on mouse Yq. We therefore also generated trees using the UPGMA algorithm, which explicitly assumes a molecular clock (Additional Files 5, 6, 7).
In this analysis, the percentage divergence of Orly from its progenitor loci (representing the date of generation of Orly) is $1.24 \%$ for Orly/Ssty1, $1.79 \%$ for Orly/Asty and $1.87 \%$ for Orly/Sly. The percentage divergence between 
Table 2: Classes of contig identified on mouse Yq

$\begin{array}{cl}\text { Huge Repeat } & \text { [GenBank:NT 161868, GenBank:NT 161875, GenBank:NT 161877, GenBank:NT 161879, GenBank:NT 161885, } \\ & \text { GenBank:NT 161892, GenBank:NT 161926, GenBank:NT 161928, GenBank:NT 165790, GenBank:NT 165791, } \\ & \text { GenBank:NT 165793, GenBank:NT 165794, GenBank:NT 165796] } \\ \text { Similar to Huge Repeat } & \text { [GenBank:NT 161923, GenBank:NT 161924, GenBank:NT 161937, GenBank:NT 165792] } \\ \text { SstylAsty-enriched } & \text { [GenBank:NT 161897, GenBank:NT 161898, GenBank:NT 161904, GenBank:NT 161919, GenBank:NT 165795, } \\ & \text { GenBank:NT 165797, GenBank:NT 165798] } \\ \text { SlylAsty-enriched } & \text { [GenBank:NT 161866, GenBank:NT 161872, GenBank:NT 161913, GenBank:NT 161916, GenBank:NT 161925] } \\ \text { Unclassifiable } & \text { [GenBank:NT 161895, GenBank:NT 161902, GenBank:NT 161906, GenBank:NT 161911] }\end{array}$

Contig classes are defined by contig content in terms of the known Yq-linked genes. The Huge Repeat consensus ordering of gene loci is shown in Figure 8.

the Orly branches of the tree (representing the date of amplification of the Huge Repeat Array) is $0.47 \%$ for the Ssty1-derived region, $0.41 \%$ for the Asty-derived region and $0.43 \%$ for the Sly-derived region. While the absolute rate of the clock cannot be determined from these data, the numbers obtained from the three trees are in good agreement with each other, strengthening our inferences of the timing of events on Yq.

\section{Conclusions of the phylogenetic study}

Taken together, these results of the phylogenetic tree analysis and locus fingerprinting of Yq contigs indicate that events on Yq occurred in the following sequence.

1) Sstx/Ssty divergence (too long ago to be addressed by nucleotide sequence analysis)

\section{2)Ssty1/Ssty2 divergence}

3) Generation of Sly by chimerism between Xmr and Xlr

4) Moderate amplification of Ssty1, Ssty2 and Asty

5) Generation of Orly by chimerism between Ssty1, Asty and $S l y$

6) Massive amplification of two familes of large-scale repeat on Yq. The first repeat family contains representatives of all Yq genes including Orly and constitutes the Huge Repeat Array, while the second specifically contains Ssty1 and Asty.

At present unresolved is the question of when the MuRVY retrovirus arrived on Yq. The presence of MuRVY-related sequence within intron 2 of every copy of Sly indicates that Sly acquired its MuRVY-derived insert in intron 2 some time between stages (3) and (6), however, the origin of MuRVY itself cannot be placed in the above sequence from available evidence.

\section{Discussion}

We report here on the genomic locus Orly and the wide variety of alternatively spliced transcripts arising from it. Orly has a complex and unusual genomic structure, being derived from partial copies of three other Yq-linked genes. Intriguingly, we also found $S l y$ to be derived by combination of existing genes, in this case a fusion of the 5 ' region of $X m r$ with the 3' region of $X l r$, together with an internal duplication of exons 3-4 of the Xmr-derived segment. This may indicate that chimerism and "exon shuffling" are a general feature of novel Y chromosome gene creation. Significantly, the two outermost partial gene loci contributing to Orly are in antisense orientation relative to each other, and retain their upstream promoter regions. We detected Orlyos transcripts in addition to Orly transcripts, and thus deduce that both promoters have retained their activity. In particular, exons $\mathrm{N} 1, \mathrm{~N} 2$ and the intervening intron are transcribed in both directions. This region derives from a MuRVY retroviral insertion into intron 2 of Sly.

There is an intriguing parallel to be drawn with the Stellate system in Drosophila melanogaster, where there is a sense/ antisense regulatory loop between X-encoded Stellate and Y-encoded Su(Ste) repeat genes [25]. In the case of Stellate, the $\mathrm{Y}$ gene arose from the $\mathrm{X}$ gene by insertion of a transposon (with active promoter) in reverse orientation $[26,27]$. Antisense Su(Ste) transcripts primed from the transposon promoter act to regulate both sense $S u(S t e)$ and Stellate transcript levels via an RNAi mechanism $[25,28]$. Similarly, Orly and Orlyos transcripts could potentially regulate each other and also Sly. A key avenue of future work is to determine the full length sequence of Orlyos, in particular whether it contains any Ssty1- or Asty-derived regions which may in turn regulate these genes.

The comparison to Stellate is especially interesting given the sex ratio skewing in male mice bearing partial Yq deletions. Partial deletions of the repressor Su(Ste) on Drosophila $\mathrm{Y}$ chromosome lead to sex ratio skewing or infertility dependent upon the X chromosomal Stellate haplotype present [29]. Stellate was hypothesised to be a 


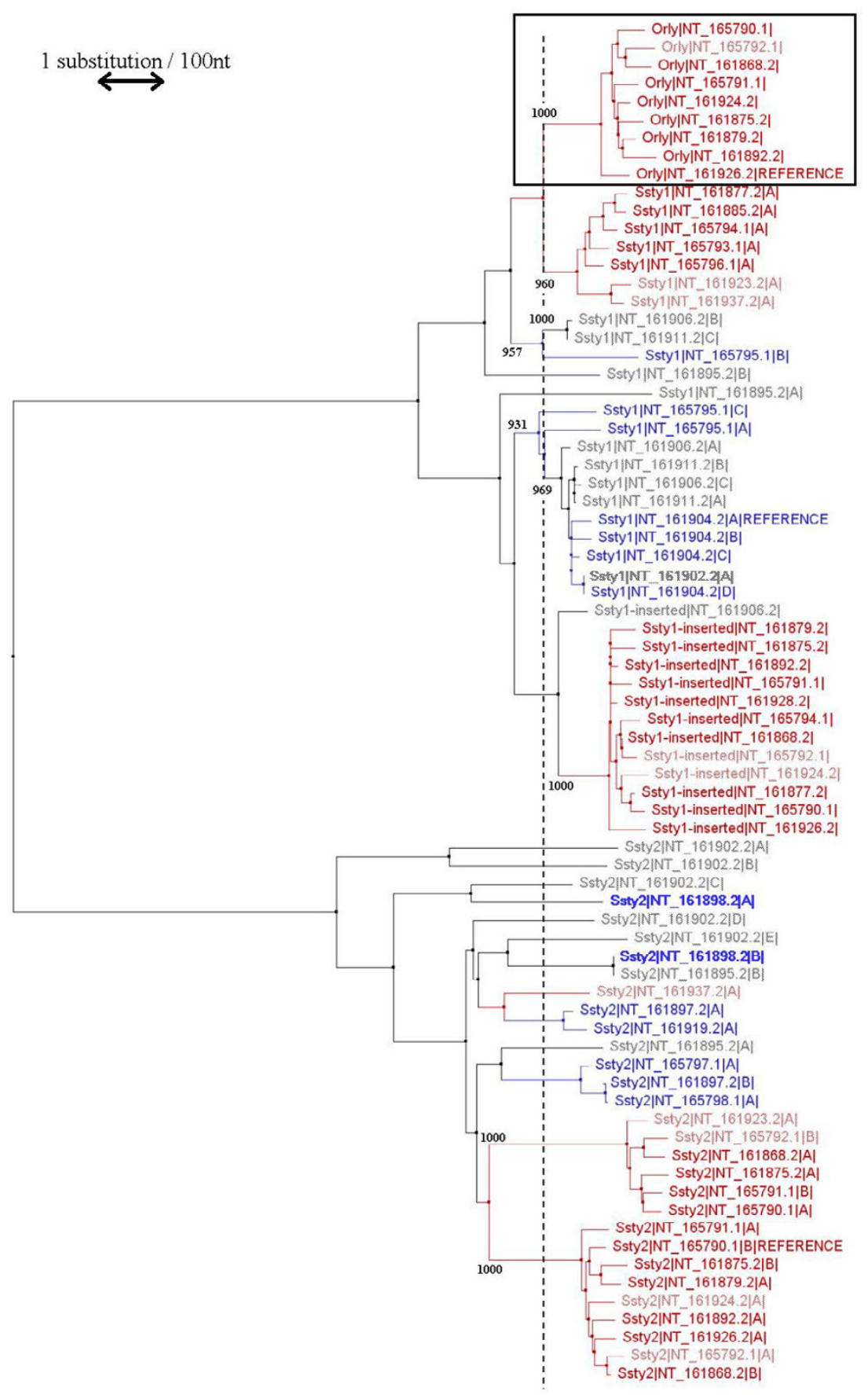

Figure 12

Phylogenetic tree of Sstyl, Ssty2 and Orly. Colours indicate which class of Yq contig each gene copy is located in (see Table 2). Red $=$ Huge Repeat contig. Pink $=$ contigs with fingerprints similar but not identical to the Huge Repeat. Green $=$ Sly/Astyenriched contigs. Blue = Ssty/Asty-enriched contigs. Grey = unclassifiable contigs. The scale bar indicates I substitution per hundred base pairs of sequence. Numbers indicate bootstrap support values (out of 1000 replicates) for key clades discussed in the text. The Orly clade is boxed. The dashed line indicates the point at which Orly was created. The intersection of this line with the branches of the tree shows how many lineages of each gene were present at the time of Orly divergence. 


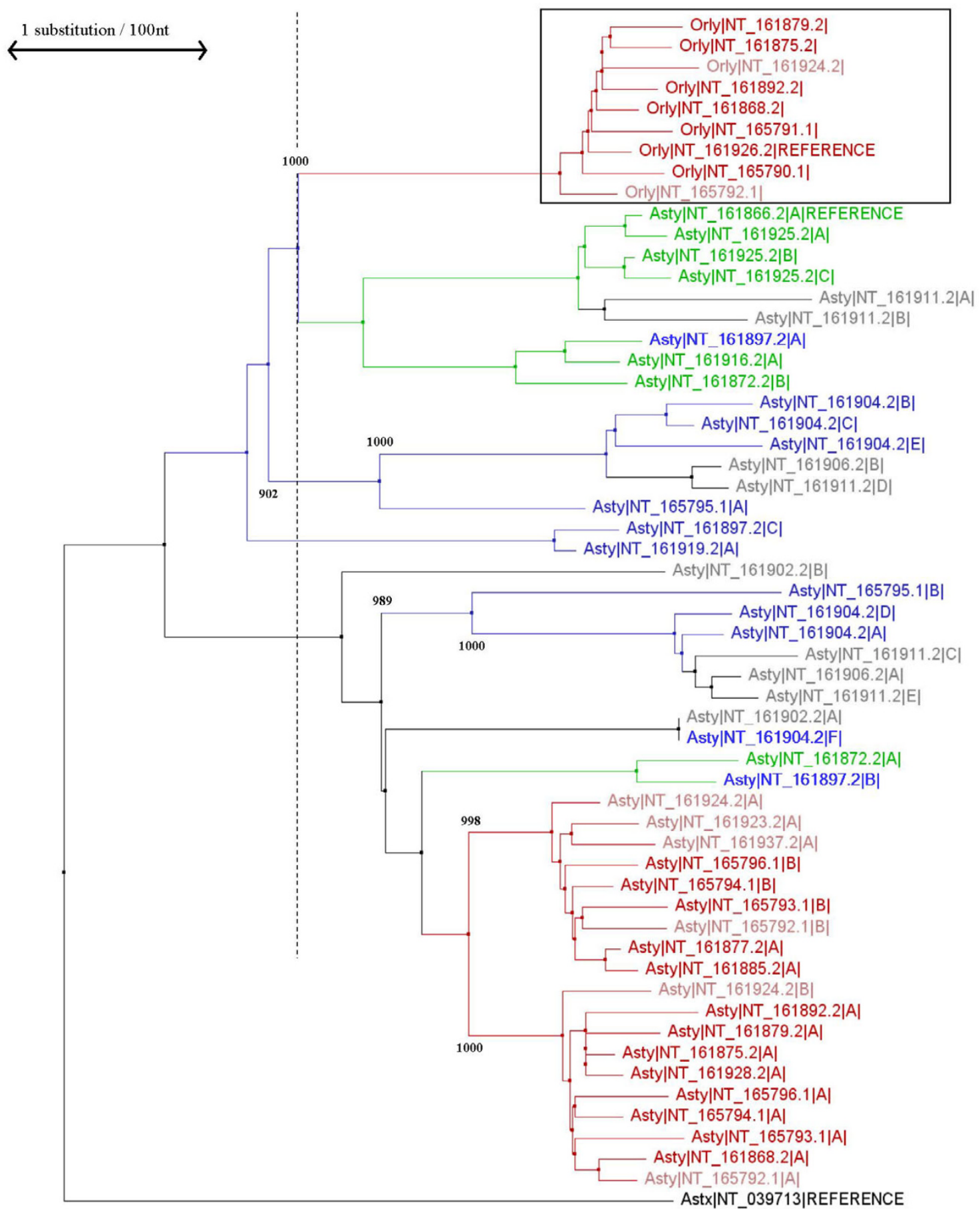

Figure 13

Phylogenetic tree of Asty and Orly. Astx is also included, and was used as the outgroup to root the tree. Colours, scale bar etc. are as in Figure $\mathbf{I} \mathbf{2}$. 


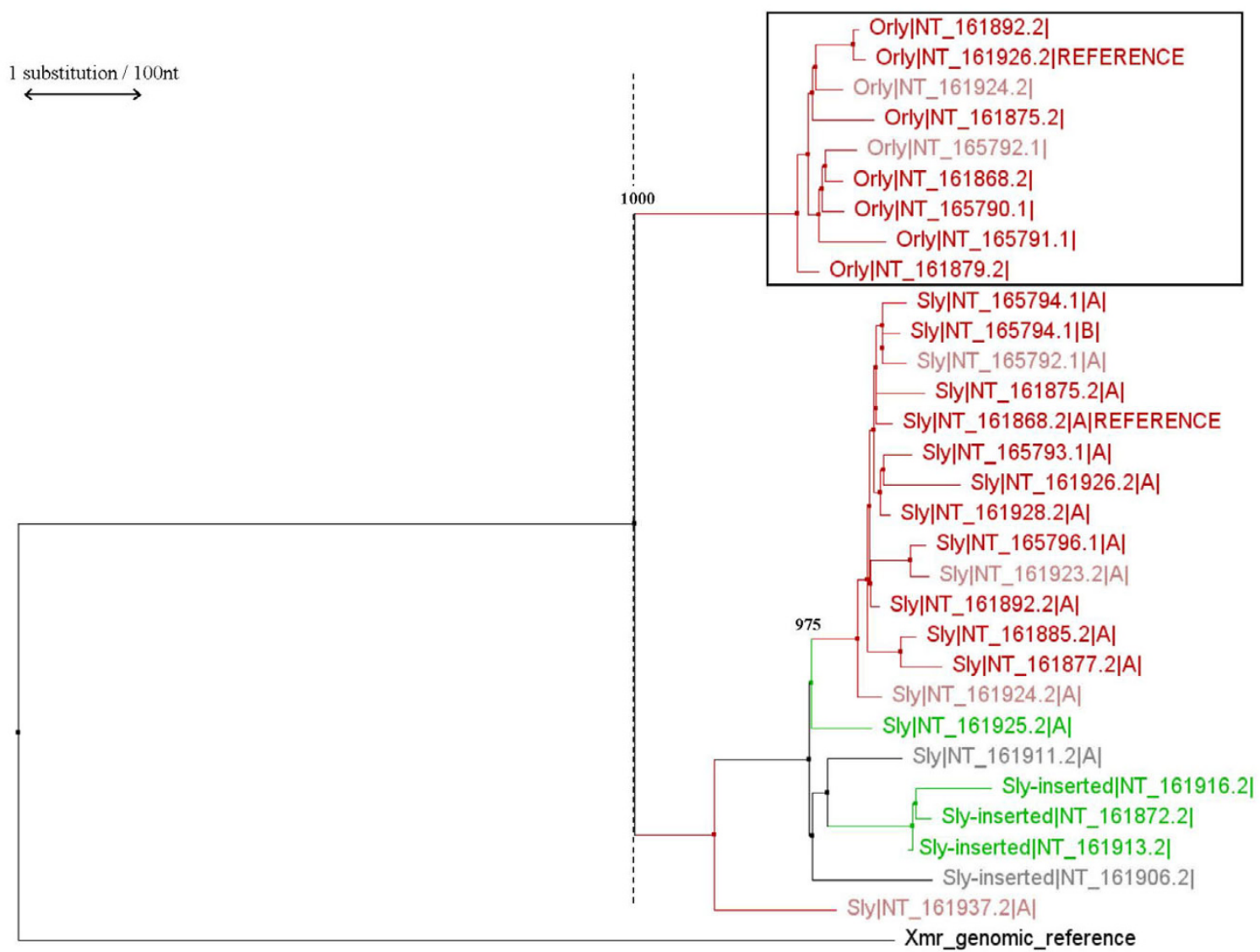

\section{Figure 14}

Phylogenetic tree of Sly and Orly. Xmr is also included, and was used as the outgroup to root the tree. Colours, scale bar etc. are as in Figure I2.

meiotic drive gene [30,31], although this is now disputed [32]. In male mice, partial deletions of Yq lead to mild teratozoospermia and sex ratio skewing $[9,11,14]$, with reduced effectiveness of Y-bearing sperm [15]. Larger deletions lead to severe teratozoospermia and infertility $[8,13]$. The mice with partial deletions show normal fertility and fecundity (in terms of number of successful matings and number of offspring per litter), thus the only effect of the decrease in Yq gene copy number appears to be the sex ratio skew.

It should be understood that the sex ratio skew in mouse with Yq deletions does not constitute meiotic drive in the classical sense, since equal numbers of $\mathrm{X}$ - and Y-bearing gametes are generated at meiosis [15]. Nevertheless, the presence of Yq-encoded genes affecting sex ratio indicates the potential for a conflict between these Yq-encoded genes and other interacting $\mathrm{X}$ - or autosomally-encoded factors. Given that Yq deletion also leads to a spermatid- specific derepression of $X$ transcripts [33], with increasing $\mathrm{X}$ gene expression correlated with the extent of the deletion, we have suggested that there may indeed be an ongoing genomic conflict between the mouse $\mathrm{X}$ and $\mathrm{Y}$ chromosomes, with $\mathrm{X}$-linked sex ratio distorter genes acting to favour generation of female offspring, and Yqlinked repressor genes acting to restore a normal 50:50 sex ratio. Such an intragenomic conflict is expected to lead to massive amplification of gene number on both chromosomes due to an "arms race" between the conflicting genes [34]. Intriguingly, the hybrid sterility seen in Mus musculus musculus/Mus musculus molossinus consomic strains is Xdependent [35].

Whether genomic conflict is involved or not, the fact that Yq-encoded genes are necessary for normal levels of $\mathrm{Y}$ chromosome transmission necessarily leads to a strong and direct evolutionary pressure to maintain the function of these genes. This may be one of the factors behind the 
recent and highly unusual gene amplification seen on mouse Yq. Orly, being composed of portions of all the other known MSYq-linked genes, must also necessarily be the most recent known addition to MSYq gene content.

\section{Conclusion}

Orly is a novel chimeric locus on mouse chromosome $\mathrm{Yq}$ which is bidirectionally transcribed, giving rise to Orly and Orlyos transcripts. These transcripts may potentially form dsRNA in partnership with each other, or with the progenitor loci Ssty1,Asty and Sly. A phylogenetic tree analysis of Yq genes indicates that Orly arose shortly prior to a massive expansion in copy number of all the Yqlinked genes. Also, potentially significantly, copies of Orly are only found in the context of the Huge Repeat Array that distinguishes MSYq - a particular segment of around $500 \mathrm{~kb}$ that appears to have been amplified en bloc. Taking the above evidence together, we propose that the emergence of Orly may have been one of the triggers that led to massive amplification of Yq sequence. Further analysis of the genomic complement of MSYq, and the copy number of the corresponding $\mathrm{X}$ genes, in a range of different mouse subspecies should help date these events more precisely, and establish whether X-Y genomic competition is a contributing factor to the gene amplifications.

\section{Methods}

Sequence comparison and detection of copies of Yq-linked genes

Nucleotide sequence alignment was performed using BLAST and ClustalW. Copies of Yq-linked genes were located within the currently-released draft sequence contigs [Mouse Chromosome Y Mapping Project (Jessica E. Alfoldi, Helen Skaletsky, Steve Rozen, and David C. Page at the Whitehead Institute for Biomedical Research, Cambridge MA, and the Washington University Genome Sequencing Center, St. Louis MO)] by pairwise alignment of reference gene sequences to each contig. All full-length hits were recorded, thus this study does not distinguish between genes and pseudogenes in each family. For Ssty1, Ssty2, Asty and Orly, a window size of 40 nt was used, while for Sly a window size of $100 \mathrm{nt}$ was used. Additional File 4 is a complete record of all loci detected in the course of this study. The reference sequences used for this search were as follows.

Ssty1: ref|NT_161904.2|MmY_159612_36:c34361-31977

Ssty2: ref|NT_165790.1|MmY_163304_36:c172129169801

Sly: ref|NT_161868.2|MmY_159576_36:275482-301680

Asty: ref|NT_161866.2|MmY_159574_36:c99035-96672
Orly:

ref|NT_161926.2|MmY_159634_36:179263194146

The reference sequences for Ssty1, Ssty2 and Sly are drawn from the Gene database of the NCBI [36]. The reference sequence for Asty was selected as the hit with the highest percentage identity to the known partial cDNA sequence [GenBank:DQ874391]. In the case of Orly, we define the locus as extending from the transcriptional start site (TSS) of the relict Ssty1 partial sequence to the TSS of the relict Sly partial sequence. The locus chosen as a reference is that encoding the known transcript Orly_v1 ([GenBank:AK015935]). Note that both the reference genome sequence and the reference gene sequences are from the C57/B16 strain. Dot plots of selected contigs and gene loci were generated using JDotter [37], with grey scale values set to highlight the appropriate homologies.

\section{Phylogenetic tree analysis}

All full-length copies of Ssty1/2, Sly, Asty and Orly identified by the contig search were used to build these trees. The reference sequences for Xmr and Astx were included in the appropriate trees in order to determine the timing of MSYq events relative to the split between $\mathrm{X}$ and $\mathrm{Y}$ homologues, however, the high degree of nucleotide sequence divergence between Sstx and Ssty precluded the inclusion of the X-linked gene for this tree.

For each gene family, a region excluding known proteincoding sequence was selected for alignment, thus nearly neutral rates of evolution can be assumed. Since Asty appears to be non-coding, the full length of all detected Asty sequences $(\sim 2.1 \mathrm{k})$ was used for the Astx/Asty/Orly tree, together with the homologous regions of Astx and Orly. For the Ssty1/Ssty2/Orly and for Xmr/Sly/Orly trees, the aligned region comprises the 3' UTR and all introns within the 3' UTR. This is $\sim 1.5 \mathrm{~kb}$ for the Ssty1/Ssty2/Orly tree and $1.4 \mathrm{k}$ for the $\mathrm{Xmr} / \mathrm{Sl} / \mathrm{Orly}$ tree.

Interestingly, the opening ATG codon was conserved in all detected copies of both families, including conservation of this codon at both ends of all copies of Orly. The significance of this observation is unclear. Alignment of the gene copies was performed using ClustalW via the EBI website [38], and ClustalX used to generate each tree using the Saitou/Nei NJ algorithm. 1000 bootstrap replicates were used to assess the robustness of each tree. Additional File 8 contains the three $\mathrm{NJ}$ trees and the ClustalW files used to generate each tree. JalView [39] was used to generate the figures included in this manuscript.

\section{RT-PCR}

RNA samples were treated for DNA contamination using the RNAse free DNAse set (Qiagen). RT-PCR was performed using the One Step RT-PCR kit (Qiagen). Briefly, 
a reverse transcription step at $50^{\circ} \mathrm{C}$ for 30 minutes was followed by an activation step at $94^{\circ} \mathrm{C}$ for 15 minutes, and then 30 cycles of PCR at $94^{\circ} \mathrm{C} / \mathrm{T}_{\mathrm{m}} / 72^{\circ} \mathrm{C}$ for $10 \mathrm{~s} / 10 \mathrm{~s} /$ $30 \mathrm{~s}$. The annealing temperature $\mathrm{T}_{\mathrm{m}}$ varied from $53-55^{\circ} \mathrm{C}$ depending on primer combination. 23 Orly partial cDNA sequences detected in this work have been submitted to GenBank, accession numbers ES316436 to ES316458.

\section{Sequencing}

Single-band RT-PCR products were purified using the Qiagen Qiaquick kit according to the manufacturers instructions. If multiple bands were present, these were gel purified using the Qiagen gel extraction kit. Purified RTPCR products were sequenced from 5' and 3' ends using standard cycle sequencing methods.

\section{Competing interests}

The author(s) declare that they have no competing interests.

\section{Additional material}

\section{Additional file 1}

Chimeric composition of the Orly locus. Dotter alignment showing the homologies between Orly and its constituent loci. Exon locations of the constituent loci are indicated, as are the novel exons contained in Orly transcripts.

Click here for file

[http://www.biomedcentral.com/content/supplementary/14712148-7-171-S1.ppt]

\section{Additional file 2}

Comparison of Sly upstream promoter region with homologous region of Orly. Annotated output from the TFSEARCH scan for potential transcription factor binding sites in the Sly upstream promoter region and the putative Orlyos promoter. Key elements such as the GCCAAT box are highlighted.

Click here for file

[http://www.biomedcentral.com/content/supplementary/1471-

2148-7-171-S2.doc]

\section{Additional file 3}

ClustalW alignment of Ssty1 and Ssty2. Annotated output from the ClustalW programme, aligning Ssty1 and Ssty2. Exons, coding region and the polyadenylation signal are highlighted for both genes.

Click here for file

[http://www.biomedcentral.com/content/supplementary/1471-

2148-7-171-S3.doc]

\section{Additional File 4}

Potential gene loci detected in Yq contigs. List of potential gene copies found within each genomic contig mapping to mouse chromosome $Y q$. Gene copies were located via BLAST comparison of reference gene loci to each contig. Start and end points (in bp) for each match are noted, as is the orientation of the potential gene copy, which may in each case be a coding gene or a pseudogene.

Click here for file

[http://www.biomedcentral.com/content/supplementary/14712148-7-171-S4.xls]

\section{Additional File 5}

UPGMA Phylogenetic tree of Ssty1, Ssty2 and Orly. Phylogenetic tree of Ssty1, Ssty 2 and Orly gene copies using the same alignment used for Figure 12. The UPGMA algorithm was used, rather than the neighbour-joining algorithm used for Figure 12.

Click here for file

[http://www.biomedcentral.com/content/supplementary/14712148-7-171-S5.ppt]

\section{Additional File 6}

UPGMA Phylogenetic tree of Asty and Orly. Phylogenetic tree of Asty and Orly gene copies using the same alignment used for Figure 13. The reference Astx sequence was used as the outgroup to root the tree. The UPGMA algorithm was used, rather than the neighbour-joining algorithm used for Figure 13.

Click here for file

[http://www.biomedcentral.com/content/supplementary/1471-

2148-7-171-S6.ppt]

\section{Additional File 7}

UPGMA Phylogenetic tree of Sly and Orly. Phylogenetic tree of Sly and Orly gene copies using the same alignment used for Figure 14. The reference Xmr sequence was used as the outgroup to root the tree. The UPGMA algorithm was used, rather than the neighbour-joining algorithm used for Figure 14.

Click here for file

[http://www.biomedcentral.com/content/supplementary/14712148-7-171-S7.ppt]

\section{Additional File 8}

Clustal alignments used for tree analysis. ZIP archive file containing the ClustalW alignments used to generate the phylogenetic trees for this project (plain text format).

Click here for file

[http://www.biomedcentral.com/content/supplementary/14712148-7-171-S8.zip]

\section{Acknowledgements}

This project was funded by the BBSRC.

\section{References}

I. Fisher R: The evolution of dominance. Biol Rev 1931, 6:345-368.

2. Charlesworth $B$ : The evolution of chromosomal sex determination and dosage compensation. Curr Biol 1996, 6:149-162.

3. Graves JA: The origin and function of the mammalian $Y$ chromosome and Y-borne genes - an evolving understanding. Bioessays 1995, 17:311-320.

4. Lahn BT, Page DC: Functional coherence of the human $\mathbf{Y}$ chromosome. Science 1997, 278:675-680.

5. Lahn BT, Pearson NM, Jegalian K: The human $\mathbf{Y}$ chromosome, in the light of evolution. Nat Rev Genet 200I, 2:207-2I6.

6. Vallender EJ, Lahn BT: How mammalian sex chromosomes acquired their peculiar gene content. Bioessays 2004, 26:159-169.

7. Ellis PJ, Affara NA: Spermatogenesis and sex chromosome gene content: an evolutionary perspective. Hum Fertil (Camb) 2006, 9(I): I-7.

8. Burgoyne PS, Mahadevaiah SK, Sutcliffe MJ, Palmer SJ: Fertility in mice requires $X-Y$ pairing and a $Y$-chromosomal "spermiogenesis" gene mapping to the long arm. Cell 1992, 71:39I-398.

9. Conway SJ, Mahadevaiah SK, Darling SM, Capel B, Rattigan AM, Burgoyne PS: Y353/B: a candidate multiple-copy spermiogenesis 
gene on the mouse $\mathbf{Y}$ chromosome. Mamm Genome 1994, 5:203-210

10. Styrna J, Imai HT, Moriwaki K: An increased level of sperm abnormalities in mice with a partial deletion of the $Y$ chromosome. Genet Res 1991, 57:195-199.

II. Styrna J, Klag J, Moriwaki K: Influence of partial deletion of the $\mathbf{Y}$ chromosome on mouse sperm phenotype. J Reprod Fertil |99|, 92:187-195.

12. Suh DS, Styrna J, Moriwaki K: Effect of $\mathbf{Y}$ chromosome and $\mathbf{H - 2}$ complex derived from Japanese wild mouse on sperm morphology. Genet Res 1989, 53:17-19.

13. Toure A, Szot M, Mahadevaiah SK, Rattigan A, Ojarikre OA, Burgoyne PS: A new deletion of the mouse $Y$ chromosome long arm associated with the loss of Ssty expression, abnorma sperm development and sterility. Genetics 2004, 166:90I-912.

14. Xian M, Azuma S, Naito K, Kunieda T, Moriwaki K, Toyoda Y: Effect of a partial deletion of $Y$ chromosome on in vitro fertilizing ability of mouse spermatozoa. Biol Reprod 1992, 47:549-553.

15. Ward MA, Burgoyne PS: The effects of deletions of the mouse Y chromosome long arm on sperm function - intracytoplasmic sperm injection (ICSI)-based analysis. Biol Reprod 2006 74(4):652-658.

16. Toure A, Clemente El, Ellis PJ, Mahadevaiah SK, Ojarikre OA, Ball PA, Reynard L, Loveland KL, Burgoyne PS, Affara NA: Identification of novel $Y$ chromosome encoded transcripts by testis transcriptome analysis of mice with deletions of the $Y$ chromosome long arm. Genome Biol 2005, 6( I 2): R I02.

17. TFSEARCH: Searching Transcription Factor Binding Sites (ver I.3) [http://www.cbrc.jp/research/db/TFSEARCH.html]

18. Gebara MM, McCarrey JR: Protein-DNA interactions associated with the onset of testis-specific expression of the mammalian Pgk-2 gene. Mol Cell Biol I992, I 2(4): |422-I43I.

19. Eicher EM, Hutchison KW, Phillips SJ, Tucker PK, Lee BK: A repeated segment on the mouse $Y$ chromosome is composed of retroviral-related, $\mathrm{Y}$-enriched and $\mathrm{Y}$-specific sequences. Genetics 1989, I 22(I): $181-192$

20. Severynse DM, Hutchison CA, Edgell MH: Identification of transcriptional regulatory activity within the 5 ' A-type monomer sequence of the mouse LINE-I retroposon. Mamm Genome 1992, 2(1):41-50.

21. RepeatMasker at NCKU Bioinformatics Center [http:// www.binfo.ncku.edu.tw/RM/RepeatMasker.php]

22. Reynard LN, Turner JM, Cocquet J, Mahadevaiah SK, Toure A, Hoog C, Burgoyne PS: Expression analysis of the mouse multi-copy $X$-linked gene XIr-related, meiosis-regulated (Xmr), reveals that Xmr encodes a spermatid-expressed cytoplasmic protein, SLX/XMR. Biol Reprod 2007, 77(2):329-335.

23. Alfoldi JE, Skaletsky H, Graves T, Minx P, Wilson RK, Page DC: Sequence of the Mouse $\mathbf{Y}$ Chromosome. Conference presentation I 8th International Mouse GenomeConference, Seattle, USA 2004.

24. Saitou N, Nei M: The neighbor-joining method: a new method for reconstructing phylogenetic trees. Mol Biol Evol 1987, 4(4):406-425.

25. Aravin AA, Naumova NM, Tulin AV, Vagin VV, Rozovsky YM, Grozder VA: Double-stranded RNA-mediated silencing of genomic tandem repeats and transposable elements in the D. melanogaster germline. Curr Biol 200I, I I ( I3): I0I7-I027.

26. Balakireva MD, Shevelyov YuYa, Nurminsky DI, Livak KJ, Gvozdev VA: Structural organization and diversification of $Y$-linked sequences comprising Su(Ste) genes in Drosophila melanogaster. Nucleic Acids Res 1992, 20( I 4):373 I-3736.

27. Kogan GL, Epstein VN, Aravin AA, Gvozdev VA: Molecular evolution of two paralogous tandemly repeated heterochromatic gene clusters linked to the $X$ and $Y$ chromosomes of Drosophila melanogaster. Mol Biol Evol 2000, I7(5):697-702.

28. Aravin AA, Klenov MS, Vagin VV, Bantignies F, Cavalli G, Gvozdev VA Dissection of a natural RNA silencing process in the Drosophila melanogaster germ line. Mol Cell Biol 2004, 24(15):742-750.

29. Palumbo G, Bonaccorsi S, Robbins LG, Pimpinelli S: Genetic analysis of Stellate elements of Drosophila melanogaster. Genetics 1994, I 38(4): I| I8I-II97.

30. Hurst LD: Is Stellate a relict meiotic driver? Genetics 1992, I 30(I):229-230.

31. Hurst LD: Further evidence consistent with Stellate's involvement in meiotic drive. Genetics 1996, I42(2):641-643.
32. Belloni M, Tritto P, Bozzetti MP, Palumbo G, Robbins LG: Does Stellate cause meiotic drive in Drosophila melanogaster? Genetics 2002, I6I(4): I55I-I559.

33. Ellis PJ, Clemente EJ, Ball P, Toure A, Ferguson L, Turner JM, Loveland $\mathrm{KL}$, Affara NA, Burgoyne PS: Deletions on mouse Yq lead to upregulation of multiple $X$ - and $Y$-linked transcripts in spermatids. Hum Mol Genet 2005, I 4(I 8):2705-27I5.

34. Partridge L, Hurst LD: Sex and conflict. Science 1998, 28 I:2003-2008.

35. Oka A, Mita A, Sakurai-Yamatani N, Yamamoto H, Takagi N, TakanoShimizu T, Toshimori K, Moriwaki K, Shiroishi T: Hybrid breakdown caused by substitution of the $X$ chromosome between two mouse subspecies. Genetics 2004, 166(2):913-924.

36. Maglott D, Ostell J, Pruitt KD, Tatusova T: Entrez Gene: genecentered information at NCBI. Nucleic Acids Res 2007:D26-31.

37. Brodie R, Roper RL, Upton C: JDotter: a Java interface to multiple dotplots generated by dotter. Bioinformatics 2004, 20(2):279-28I.

38. EBI Tools: ClustalW [http://www.ebi.ac.uk/clustalw/index.html]

39. Clamp M, Cuff J, Searle SM, Barton GJ: The Jalview Java alignment editor. Bioinformatics 2004, 20(3):426-427.
Publish with Bio Med Central and every scientist can read your work free of charge

"BioMed Central will be the most significant development for disseminating the results of biomedical research in our lifetime. "

Sir Paul Nurse, Cancer Research UK

Your research papers will be:

- available free of charge to the entire biomedical community

- peer reviewed and published immediately upon acceptance

- cited in PubMed and archived on PubMed Central

- yours - you keep the copyright
BioMedcentral 